\title{
Preparation of Platinum Catalysts on Porous Titanium Nitride Supports by Atomic Layer Deposition and Their Catalytic Performance for Oxygen Reduction Reaction
}

\author{
Xiaolong Tang ${ }^{1}$, Shenghui Zhang ${ }^{1}$, Jing Yu ${ }^{1}$, Chunxiao Lü ${ }^{1}$, Yuqing Chi ${ }^{1}$, Junwei Sun ${ }^{1}$, \\ Yu Song ${ }^{1}$, Ding Yuan ${ }^{1,2, *}$, Zhaoli Ma ${ }^{1}$, Lixue Zhang ${ }^{1, *}$ \\ ${ }^{1}$ College of Chemistry and Chemical Engineering, Chemical Experimental Teaching Center, Qingdao University, Qingdao 266071, \\ Shandong Province, P. R. China. \\ ${ }^{2}$ Industrial Research Institute of Nonwovens \& Technical Textiles, College of Textiles \& Clothing, Qingdao University, \\ Qingdao 266071, Shandong Province, P. R. China.
}

\begin{abstract}
The exploitation of high-performing stable oxygen reduction reaction (ORR) electrocatalysts is critical for energy storage and conversion technologies. The existing highefficiency electrocatalysts applied to the ORR are mainly based on Pt and its alloys. Moreover, carrier catalysts are the most widely used in actual electrocatalysis. A suitable carrier not only improves the utilization rate of precious metals and the service life of the catalyst, but also serves as a co-catalyst to ameliorate the catalytic activity through a synergistic effect in the reaction. Therefore, research into Pt-based electrocatalysts mainly focuses on the precious metal Pt and

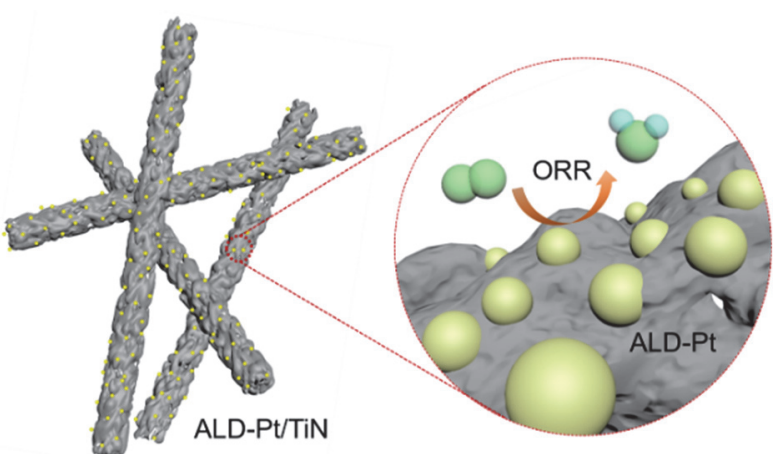
the carrier. With the aim of improving the activity and durability of Pt-based catalysts for the ORR, one-dimensional porous titanium nitride (TiN) nanotubes with a large specific surface area as well as good conductivity, electrochemical stability, and corrosion resistance were prepared in this study, and then, Pt nanoparticles were deposited on the TiN-support by atomic layer deposition (ALD). ALD is a novel and simple method for the preparation of films or nanoparticles with fine control of the thickness or size, respectively. The results of X-ray diffraction (XRD) and high-resolution transmission electron microscopy (HR-TEM) experiments confirmed that the Pt nanoparticles obtained by ALD (ALD-Pt/TiN) were face-centered cubic (fcc) crystals with a uniform size and were highly dispersed on the surface of TiN. X-ray spectroscopy (XPS) measurements verified that the binding energy of $\mathrm{Pt} 4 f$ in ALD-Pt/TiN was positively shifted by $0.33 \mathrm{eV}$ with respect to that of the $\mathrm{Pt} / \mathrm{C}$ catalyst, indicating strong electronic interactions between the ALD-Pt nanoparticles and the TiN carriers. Linear sweep voltammetry (LSV) and cyclic voltammetry (CV) analyses revealed that ALD-Pt/TiN possessed high activity for the ORR and favorable durability. The onset potential and diffusion-limiting current density of ALD-Pt/TiN were similar to those of commercial $\mathrm{Pt} / \mathrm{C}$, while the half-wave potential was $20 \mathrm{mV}$ higher than that of commercial $\mathrm{Pt} / \mathrm{C}$, indicating better electrocatalytic performance of the designed material. Furthermore, the electrocatalytic mechanism and kinetics for ALD$\mathrm{Pt} / \mathrm{TiN}$ were investigated by rotating ring-disc electrode (RRDE) experiments. The results suggested that the electron transfer number of the ALD-Pt/TiN catalyst was about 3.93, indicating that the ORR on the electrode was dominated by an efficient four-electron pathway. At the same time, the peroxide content was only $5 \%$. The results of accelerated durability testing (ADT) showed that ALD-Pt/TiN had better ORR stability than Pt/C. This excellent electrocatalytic performance was probably due to the high dispersibility of the Pt nanoparticles deposited by ALD, good conductivity and corrosion resistance of TiN, and strong interactions between ALD-Pt and the TiN support. This work provides a reliable strategy for the design
\end{abstract}

Received: June 24, 2019; Revised: August 15, 2019; Accepted: August 22, 2019; Published online: August 30, 2019.

*Corresponding authors. Emails: zhanglx@qdu.edu.cn (L.Z.); yuanding@qdu.edu.cn (D.Y.). Tel.: +86-532-85953981 (L.Z.); +86-532-85957336 (D.Y.). The project was supported by the National Natural Science Foundation of China (21775078, 21802079), Shandong Provincial Natural Science Foundation, China (ZR2016JL007), Postdoctoral Science Foundation of China (2018M642605). 
of new electrocatalytic materials with high activity and stability. Future research will focus on the strong interactions between ALD-Pt and the TiN carriers.

Key Words: Porous Titanium Nitride; Platinum nanoparticles; Atomic layer deposition; Electrocatalysis; Oxygen reduction reaction

\section{多孔氮化钛载体上铂催化剂的原子层沉积制备及其催化氧气还原性能}

唐小龙 ${ }^{1}$, 张盛辉 ${ }^{1}$, 于婧 ${ }^{1}$, 吕春晓 ${ }^{1}$, 迟雨晴 ${ }^{1}$, 孙君伟 ${ }^{1}$, 宋誉 ${ }^{1}$, 袁丁 ${ }^{1,2,{ }^{*}}$, 马兆立 ${ }^{1}$, 张立学 1 , $^{*}$

1 青岛大学化学化工学院, 化学实验教学中心, 山东青岛 266071

2 青岛大学非织造材料与产业用纺织品创新研究院, 纺织服装学院, 山东 青岛 266071

摘要: 为有效解决铂 $(\mathrm{Pt})$ 催化剂用于氧气还原反应 $(\mathrm{ORR})$ 面临的催化活性及稳定性问题, 本文首先合成了具有良好导电 性、电化学稳定以及耐腐蚀等优点的一维多孔氮化铁(TiN)纳米管载体材料, 然后使用原子层沉积技术(ALD)在TiN载体上 沉积制备了Pt催化剂(ALD-Pt/TiN), 所得的Pt纳米颗粒尺寸均匀、高度分散且与TiN载体之间存在着较强的相互作用。催 化氧气还原活性和稳定性测试表明, 所得的ALD-Pt/TiN对ORR具有较高的催化活性, 同时兼具良好的稳定性和耐久性。 相比商用Pt/C, ALD-Pt/TiN的起始电位和稳态极限电流密度与其相近, 半波电位则高出了 $20 \mathrm{mV}$, 表现出优异的电催化 性能。其优良的电催化性能主要归因于ALD沉积Pt纳米颗粒的高分散性, 一维多孔结构TiN载体的高比表面积、良好导电 性和抗腐蚀性能以及ALD-Pt与TiN载体间较强的相互作用等综合影响。本工作为设计新型高催化活性、高稳定性的Pt基 催化剂提供了有益借鉴。

关键词: 多孔氮化铁; Pt纳米颗粒; 原子层沉积; 电催化; 氧气还原反应 中图分类号: 0646

\section{1 引言}

寻找高效、安全并且环境友好的可再生能源 已经成为人类社会可持续发展的重要突破口, 受 到科研工作者的高度关注 ${ }^{1,2}$ 。燃料电池与金属-空 气电池作为新型电化学能源转化与存储技术吸引 了广泛研究 ${ }^{3-6}$ 。其中, 能量输出过程中重要组成一 阴极氧还原反应(ORR), 其反应能垒较高、动力学 过程缓慢, 须使用催化剂降低反应能垒、减小电化 学极化、加快反应速率7,8。铂 $(\mathrm{Pt})$ 基催化剂, 如碳 载 $\mathrm{Pt}(\mathrm{Pt} / \mathrm{C})$ 或 $\mathrm{Pt}$ 合金 $(\mathrm{Pt}-\mathrm{M} / \mathrm{C})$, 便能够有效加速电极 上的ORR过程 ${ }^{9-12}$ 。这主要归因于Pt原子与氧的键 能适中, 既能够打断 $\mathrm{O}=\mathrm{O}$ 双键, 又可使表面吸附 的氧物种后续反应生成水, 因此表现出较高的电 催化氧还原活性 9 。目前, 研究人员探索多种不同 方法制备Pt基催化剂, 通过合成手段的优化提升 Pt 基催化剂的ORR催化活性和利用率 ${ }^{13}$ 。其中, 原子 层沉积技术(ALD) 是一种重要的功能性薄膜/颗粒 材料制备新技术，该技术通过控制连续的自限制 性表面半反应, 可在纳米级尺度上精确控制材料 的成分、形貌和尺寸等 ${ }^{44,15}$ 。ALD可以通过调控沉 积参数和循环次数, 精确控制催化剂颗粒的粒径,
同时保持颗粒的高分散性，提高催化剂利用率 ${ }^{15}$ 。 近些年，多类使用ALD技术制备的负载型金属纳 米催化剂相继被报道 ${ }^{16-19}$ 。研究表明, 利用ALD技 术制备Pt基纳米催化剂(ALD-Pt), 具有精细可控、 简单易行等优势, 对于优化催化剂性能有重要意 $义^{19}$ 。

现阶段, 实际电催化应用中一般使用载体型 催化剂 ${ }^{9}$ 。合适的载体不仅能够提高贵金属的利用 率和催化剂的稳定性, 还可以作为助催化剂, 在 催化反应过程中通过协同效应来增强催化剂的 活性 ${ }^{20,21}$ 。但当前商品化 $\mathrm{Pt} / \mathrm{C}$ 催化剂所用碳载体的 稳定性不佳也严重限制了其实际应用 $22-24$ 。过渡金 属氮化物, 如氮化钢 $(\mathrm{TiN})$, 是氮原子进入过渡金 属的晶格而形成的一类具有金属性质的间充化合 物, 表现出高导电性、耐腐蚀性、耐高温性以及高 化学稳定性等优点, 有望成为电催化剂的高性能 载体 ${ }^{25-27}$ 。研究结果显示, TiN载体比碳载体具有 更强的抗电化学腐蚀性能, 能够提高催化剂的 ORR活性、稳定性和耐久性。

本文中，首先合成了一种具有高比表面积、良 好导电性和抗腐蚀性能的一维多孔结构 TiN材料 
作为载体, 继而采用 ALD方法在其上沉积 Pt纳米 颗粒, 制备出具备优异ORR活性和耐久性的ALD$\mathrm{Pt} / \mathrm{TiN}$ 催化剂。根据透射电子显微镜(TEM)、X射 线衍射 $(X R D)$ 和X射线光电子能谱(XPS)等相关数 据推论, 该优良的电催化性能主要由于ALD沉积 $\mathrm{Pt}$ 纳米颗粒的高分散性、一维多孔结构TiN载体的 高比表面积、良好的导电性和抗腐蚀性能以及 ALD-Pt与 TiN载体间较强的相互作用等共同影响。

\section{2 实验部分}

\subsection{ALD-Pt/TiN催化剂的制备}

实验所用的 (三甲基) 甲基环戊二烯合铂 (MeCpPtMe3, 99\%), Nafion溶液 $5 \%$ ( $w$, 质量分 数) $)$ 和二氧化钛颗粒 $\left(\mathrm{TiO}_{2}\right.$, 锐钛矿型, 325 目)购自 Sigma-Aldrich; 无水乙醇 $\left(\mathrm{C}_{2} \mathrm{H}_{5} \mathrm{OH}\right)$ 、氢氧化钠 $(\mathrm{NaOH})$ 和硫酸 $\left(\mathrm{H}_{2} \mathrm{SO}_{4}\right)$ 购自国药集团化学试剂有 限公司, 均为分析纯; 商用 $\mathrm{Pt} / \mathrm{C}$ 催化剂 $(20 \%(w))$ 购 自上海迈瑞尔化学技术有限公司; 高纯氮气 (99.999\%)、高纯氧气 $(99.999 \%)$ 以及高纯氨气 (99.999\%)购自青岛德海伟业科技有限公司; 实验 过程中使用超纯水的电阻率为 $18.2 \mathrm{M} \Omega \cdot \mathrm{cm}$ 。

钛酸盐纳米管、 $\mathrm{TiO}_{2}$ 和 $\mathrm{TiN}$ 的制备: 称取 $6 \mathrm{~g}$ 二 氧化钛颗粒于 $80 \mathrm{~mL} 、 10 \mathrm{~mol} \cdot \mathrm{L}^{-1} \mathrm{NaOH}$ 的溶液中 超声分散均匀后, 转移至反应釜内, 放入烘箱中于 $150{ }^{\circ} \mathrm{C}$ 反应 $48 \mathrm{~h}$ 后得到钛酸盐纳米管 ${ }^{28}$ 。之后, 将 产物离心清洗、烘干备用。取上步合成出的钛酸盐 纳米管材料置于马弗炉(KSL-1200X, 合肥科晶材 料技术有限公司)中, $500^{\circ} \mathrm{C}$ 热处理 $2 \mathrm{~h}$ 后得到纳米 管状 $\mathrm{TiO}_{2}$ 。随后, 称取适量 $\mathrm{TiO}_{2}$ 放入管式炉 $(\mathrm{OTF}-$ $1200 \mathrm{X}$, 合肥科晶材料技术有限公司)中, 以氨气作 为氮化气体氛围, $800^{\circ} \mathrm{C}$ 热处理 $2 \mathrm{~h}$, 即得到 TiN纳 米管材料。

TiN上Pt催化剂的ALD制备: 称取 $10 \mathrm{mg}$ TiN粉 末于 $500 \mu \mathrm{L}$ 乙醇 $/ 500 \mu \mathrm{L}$ 超纯水的混合溶液中超声 分散30 min, 然后均匀滴涂在载玻片。待干燥后, 将该载玻片置于高温封闭式ALD反应器(D100$4 \mathrm{P} 8 \mathrm{C} 8 \mathrm{H} 2 \mathrm{~F}$, 重庆诺图科技有限公司)的反应腔中 进行原子层沉积。在反应过程中, 以 $\mathrm{MeCpPtMe}_{3}$ 和 臭氧 $\left(\mathrm{O}_{3}\right)$ 作为 $A L D$ 制备 $\mathrm{Pt}$ 催化剂的前驱物, 高纯氮 气作为载气、吹扫气体和保护气体 ${ }^{19}$ 。其中, $\mathrm{O}_{3}$ 由 高纯氧通过臭氧发生器制备(青岛国林实业有限 责任公司), 既有利于在载体表面形成更多的活性 位点, 同时作为氧元素的来源。在典型的ALD单次 循环操作中, $\mathrm{MeCpPtMe}_{3}$ 前驱体的脉冲、曝光和清 洗时间分别为 $1 、 2$ 和 $2 \mathrm{~s}, \mathrm{O}_{3}$ 分别为 $1 、 10$ 和 $5 \mathrm{~s}$ 。实 验操作循环次数分别为 $30 、 50$ 和 70 个完整的ALD
循环反应。最后, 取出载玻片, 将粉末刮下, 收集 备用。

\section{2 催化剂的结构表征}

所制备材料的晶形结构通过 X射线衍射仪 (LabX XRD-6100, 日本岛津仪器公司, 日本)表征, 利用X射线光电子能谱仪(ESCALAB MK II型, 英 国VG科学仪器公司, 英国)对材料的表面组成和元 素价态等进行分析, 采用透射电子显微镜 (HITACHI H-8100, 日本日立公司, 日本)观察催 化剂的形貌和结构。

\section{3 催化剂的电化学表征}

催化剂的催化氧气还原活性和稳定性使用线 性扫描伏安法(LSV)和循环伏安法 $(\mathrm{CV})$ 等方法进 行评估。测试装置为法国Bio-logic VSP-300电化学 工作站与日本ALS 的 RRDE-3A型旋转圆盘电极 (直径 $0.4 \mathrm{~cm}$, 面积为 $0.1256 \mathrm{~cm}^{2}$ )。其中, 参比电极 为 $\mathrm{Ag} / \mathrm{AgCl}$ 电极 (内置饱和 $\mathrm{KCl}$ 溶液), 对电极为铂 丝, 涂有催化剂层的旋转圆盘电极为工作电极。本 文中所使用的电位值, 根据公式 $E(v s \mathrm{RHE})=E(v s$ $\mathrm{Ag} / \mathrm{AgCl})+0.256 \mathrm{~V}$, 均转换为可逆氢电极电位; 电流密度为电流与电极面积之比。

制备测试电极的过程如下: 称取 $10 \mathrm{mg}$ ALD$\mathrm{Pt} / \mathrm{TiN}$ 加入含 $20 \mu \mathrm{L}$ Nafion (5\% (w)) 溶液/750 $\mu \mathrm{L}$ 乙 醇 $/ 250 \mu \mathrm{L}$ 超纯水的混合溶液, 超声分散均匀后, 使 用移液枪移取 $8.6 \mu \mathrm{L}$ 滴涂在旋转圆盘电极表面, 干 燥后进行电化学测试。

电化学表征: 分别在 $\mathrm{N}_{2}$ 饱和与 $\mathrm{O}_{2}$ 饱和的 0.5 $\mathrm{mol} \cdot \mathrm{L}^{-1} \mathrm{H}_{2} \mathrm{SO}_{4}$ 溶液中进行 $\mathrm{CV}$ 测试, 其扫描电势范 围为 $0.05-1.2 \mathrm{~V}$ (vs RHE), 扫描速率为 $50 \mathrm{mV} \cdot \mathrm{s}^{-1}$ 。 随后, 在 $\mathrm{O}_{2}$ 饱和的 $0.5 \mathrm{~mol} \cdot \mathrm{L}^{-1} \mathrm{H}_{2} \mathrm{SO}_{4}$ 中进行 LSV测 试, 测试扫描范围为 $0.05-1.2 \mathrm{~V}$ ( vs RHE), 扫描速 率为 $10 \mathrm{mV} \cdot \mathrm{s}^{-1}$ 。为研究ORR电催化机理和动力学, 将转速设置为 $400 、 625 、 900 、 1225 、 1600$ 和 2025 $\mathrm{r} \cdot \mathrm{min}^{-1}$ 进行LSV测量。

使用旋转环盘电极(RRDE) 测试计算电子转移 数和过氧化氢 $\left(\mathrm{H}_{2} \mathrm{O}_{2}\right)$ 产率, 测试参数与旋转圆盘电 极(RDE)实验相同。加速老化测试(ADT)是评价催 化剂电催化稳定性的重要研究手段, 本文中ADT 测试是在空气氛围中, 于 $0.5 \mathrm{~mol} \cdot \mathrm{L}^{-1} \mathrm{H}_{2} \mathrm{SO}_{4}$ 进行 $\mathrm{CV}$ 扫描, 电势扫描范围是 $0.05-1.2 \mathrm{~V}$ ( vs RHE), 循环 圈数为 5000 圈, 扫描速率为 $50 \mathrm{mV} \cdot \mathrm{s}^{-1}$ 。

\section{3 结果与讨论}

本文利用热处理钛酸盐和随后的氮化反应制 备耐腐蚀性的 TiN作为 ORR 催化剂载体。图1为制 备的 $\mathrm{TiO}_{2}$ 、 $\mathrm{TiN}$ 及 ALD-Pt/TiN的XRD图谱。其中, 


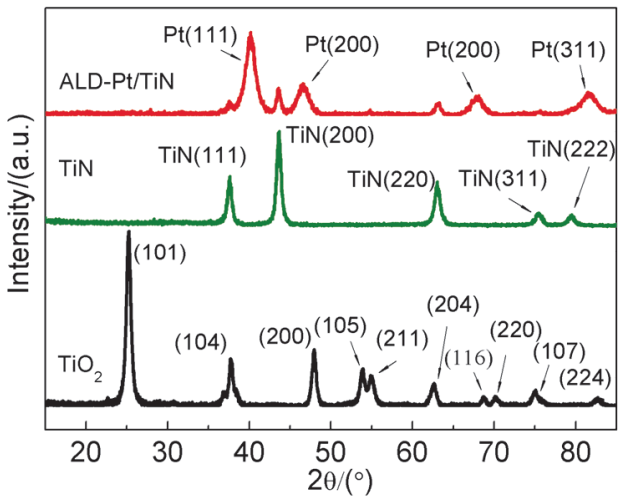

图 1 ALD-Pt/TiN、TiN 以及 $\mathrm{TiO}_{2}$ 的 XRD 图谱

Fig. 1 XRD patterns of ALD-Pt/TiN, TiN and $\mathrm{TiO}_{2}$.

$\mathrm{TiO}_{2}$ 衍射峰的位置证实其晶相属于锐钛矿型 (JCPDS No. 21-1272), 而 TiN 和 ALD-Pt/TiN 在 $36.7^{\circ} 、 61.8^{\circ} 、 74.1^{\circ}$ 和 $77.9^{\circ}$ 的衍射峰, 表明制备的 TiN为面心立方结构(JCPDS No. 38-1420) ${ }^{25,27}$ 。同 时, XRD并未检测出其他杂质峰, 表明在氮化处理 过程中, $\mathrm{TiO}_{2}$ 完全转化为 TiN。ALD-Pt/TiN位于 $39.8^{\circ} 、 46.2^{\circ}$ 和 $67.6^{\circ}$ 的衍射峰值分别属于面心立 方结构(fcc)Pt的(111)、(200)和(220)晶面, 证实Pt成 功负载在氮化钛上 ${ }^{15,17}$ 。

通过TEM进一步分析所制备材料的微观形貌 和结构(图2)。由图 $2 \mathrm{a}$ 可知, $\mathrm{TiO}_{2}$ 纳米管的直径均 匀, 约 $10 \mathrm{~nm}$ 左右。经过高温氮化过程得到的 TiN 为多孔一维支架状、由不同粒径纳米材料组成的 团聚体(图2b)。简单来说, 在氮化过程中, $\mathrm{TiO}_{2}$ 发 生断裂和重新排列, 形成多孔的中空TiN纳米管状

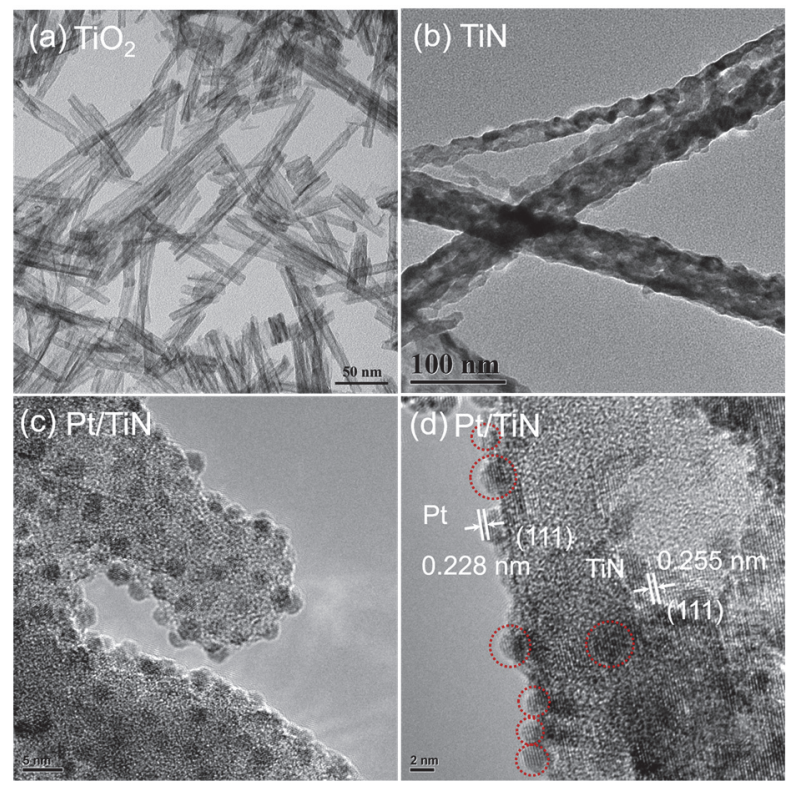

图 $2 \mathrm{TiO}_{2}(\mathrm{a}) 、 \mathrm{TiN}(\mathrm{b}) 、 \mathrm{ALD}-\mathrm{Pt} / \mathrm{TiN}(\mathrm{c}, \mathrm{d})$ 的 TEM 图

Fig. 2 TEM images of $\mathrm{TiO}_{2}$ (a), $\mathrm{TiN}(\mathrm{b})$,

ALD-Pt/TiN (c, d).
结构 27 。该一维多孔结构, 具有大的比表面积, 能 够在反应过程中提高传质速率。此外, 一维 TiN纳 米管相互交织具有良好的导电性, 可以为电子传 导提供通路 ${ }^{27}$ 。通过交流阻抗测试进一步证实, ALD50-Pt/TiN 催化剂与商品化 $\mathrm{Pt} / \mathrm{C}$ 的导电性接 近, 展现出良好的电子传输性能 (见图 S1, Supporting Information (SI))。图2c, d为ALD循环 沉积 50圈的ALD-Pt/TiN的TEM图, 可以看出Pt以 独立颗粒状负载在TiN上, 没有团聚现象。通过高 分辨率TEM图测量到间距为 $0.255 \mathrm{~nm}$ 的晶面, 对应 于面心立方结构 $\mathrm{TiN}$ 晶体的(111)晶面, 如图 $2 \mathrm{~d}$ 所 示。同时, 发现间距为 $0.228 \mathrm{~nm}$ 的晶面, 归属于 $\mathrm{Pt}$ 的(111)晶面, 上述分析结果与XRD结果相一致。 此外, 通过对比采用不同ALD循环周期数(30圈、 50 圈与70圈)制备的ALD-Pt/TiN样品的TEM图像, 发现ALD-Pt在TiN上皆分布均匀, 且随着ALD沉 积周期数的增大, $\mathrm{Pt}$ 纳米颗粒的尺寸稍有所增加 (见图 S2 (SI)), 其中ALD50-Pt/TiN的Pt纳米颗粒平 均尺寸约 $2.3 \mathrm{~nm}$ 。

为研究ALD-Pt与 TiN载体之间相互作用, 对 ALD-Pt/TiN和商用 $\mathrm{Pt} / \mathrm{C}$ 进行了 XPS 测试。图 3 为 ALD-Pt/TiN和商用 $\mathrm{Pt} / \mathrm{C}$ 中 $\mathrm{Pt}$ 的 $4 f$ 电子轨道结合能 图。经过分峰拟合后, Pt 4f峰得出 $\operatorname{Pt}(0) 、 \operatorname{Pt}(\mathrm{II})$ 两个 峰。对比商用 $\mathrm{Pt} / \mathrm{C}, \mathrm{ALD}-\mathrm{Pt} / \mathrm{TiN}$ 催化剂的峰发生约 $0.33 \mathrm{eV}$ 的正移, 意味着 $\mathrm{Pt}$ 原子与 $\mathrm{Ti}$ 或 $\mathrm{N}$ 原子之间存 在电荷的转移, 即 $\mathrm{Pt}$ 与 TiN载体间有很强的电子相 互作用 21,22 。

接下来, 在 $0.5 \mathrm{~mol} \cdot \mathrm{L}^{-1} \mathrm{H}_{2} \mathrm{SO}_{4}$ 溶液中对样品进 行了线性极化曲线 $\left(1600 \mathrm{r} \cdot \mathrm{min}^{-1}\right)$ 的测试, 以评估 ALD-Pt/TiN和商用 Pt/C 催化剂氧还原催化性能的 差异。从图 $4 a$ 可看出, ALD-Pt/TiN对ORR具有优异 的催化活性。其中, ALD50-Pt/TiN、ALD70-Pt/TiN 的起始电位与商品化 $\mathrm{Pt} / \mathrm{C}$ 相近, 并且 ALD50-

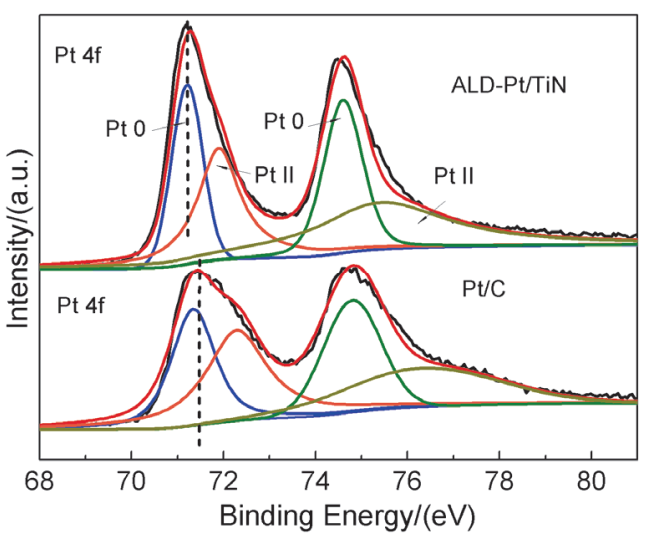

图 $3 \mathrm{ALD}-\mathrm{Pt} / \mathrm{TiN}$ 和 Pt/C 的 XPS 图谱

Fig. 3 XPS spectra of Pt/C and ALD-Pt/TiN. 

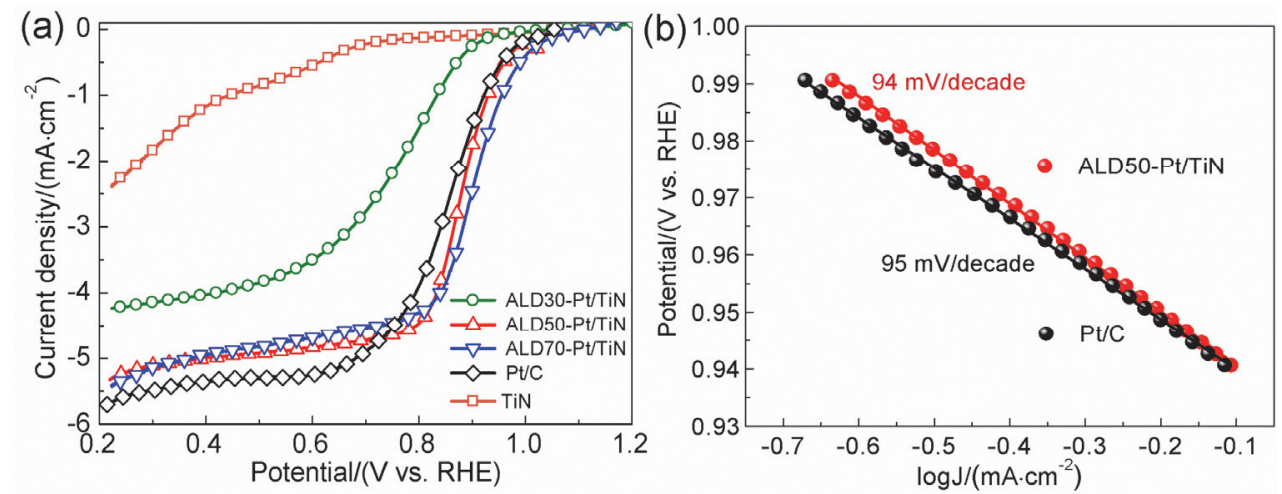

图 $4 \mathrm{ALD}-\mathrm{Pt} / \mathrm{TiN} 、 \mathrm{Pt} / \mathrm{C}$ 和 TiN 在氧气饱和的 $0.5 \mathrm{~mol} \cdot \mathrm{L}^{-1} \mathrm{H}_{2} \mathrm{SO}_{4}$ 溶液中的 $\mathrm{ORR}$ 极化曲线(a)和塔菲尔曲线 (b),

转速为 $1600 \mathrm{r} \cdot \mathrm{min}^{-1}$, 扫描速率为 $10 \mathrm{mV} \cdot \mathrm{s}^{-1}$

Fig. 4 The ORR polarization curves (a) and the corresponding Tafel plots (b) of ALD-Pt/TiN, Pt/C and TiN at $1600 \mathrm{r} \cdot \mathrm{min}^{-1}$ rotation and $10 \mathrm{mV} \cdot \mathrm{s}^{-1}$ scan rate in $0.5 \mathrm{~mol} \cdot \mathrm{L}^{-1} \mathrm{H}_{2} \mathrm{SO}_{4}$ solution saturated with $\mathrm{O}_{2}$.

$\mathrm{Pt} / \mathrm{TiN}$ 的半波电位高出约 $20 \mathrm{mV}$, 表现出优异的 ORR催化性能。推测可能是由于一维TiN纳米材料 的导电性能较好、比表面积大、Pt纳米颗粒分散均 匀以及Pt电子结构的变化等多方面影响 27 。在电催 化过程中，电子的传输速度和催化活性位点的暴 露对氧还原性能有很大的影响 29 。此外, 有研究表 明 $\mathrm{OH}$ 基团能够吸附在TiN表面, 并与邻近Pt原子表 面上的OH基团产生排斥作用，降低Pt表面OH的覆 盖率 $21,30,31$ 。Pt原子与含氧物种间的相互作用减弱、 $\mathrm{OH}$ 基团覆盖率的减少有助于 ORR反应过程中吸 附氧的解离以及产物的移除 ${ }^{13,32}$, 这可能也是 $\mathrm{ALD}-\mathrm{Pt} / \mathrm{TiN}$ 具有与商用 $\mathrm{Pt} / \mathrm{C}$ 相近 $\mathrm{ORR}$ 性能的原因 之一。除此之外, 不同ALD循环周期数制备的 ALD-Pt/TiN 的 ORR 催化性能之间存在差异, ALD50-Pt/TiN与ALD70-Pt/TiN催化性能相当, 都 明显优于ALD30-Pt/TiN，综合考虑到提高贵金属 利用率, 降低成本等因素, 后续选取ALD50-Pt/TiN 作为研究对象。

ALD50-Pt/TiN和Pt/C的塔菲尔曲线, 如图4b所 示, 也证实了ALD-Pt/TiN具有优异的催化活性。在 电压范围为 0.94 至 $0.99 \mathrm{~V}$ 区间, ALD50-Pt/TiN的塔 菲尔斜率与 $\mathrm{Pt} / \mathrm{C}$ 的塔菲尔斜率接近, 说明氧还原反 应动力学与商用 $\mathrm{Pt} / \mathrm{C}$ 相似 ${ }^{33,34}$, 具有实际应用的潜 力。综上, 可以合理推测ALD-Pt/TiN的多孔一维结 构、高比表面积及其表面高分散的Pt以及因 Pt/TiN 之间强相互作用而影响Pt电子结构的变化等所产 生的协同作用，使其具备了与商业 $\mathrm{Pt} / \mathrm{C}$ 媲美的 ORR催化活性。

通过LSV 方法在RDE和RRDE上进一步研究 $\mathrm{ALD} 50-\mathrm{Pt} / \mathrm{TiN}$ 的电催化机理和动力学。图 5 为 ALD50-Pt/TiN 在不同旋转速度下获得的 LSV 曲 线, 极限电流密度随着转速增加而增加, 表明其
$\mathrm{ORR}$ 电催化反应受到溶液中溶解 $\mathrm{O}_{2}$ 向催化剂表面 的扩散控制 35 。通过拟合得到的Koutecky-Levich (K-L)曲线(如图 5插图)可知ALD50-Pt/TiN在不同 电压下的K-L曲线均表现出良好的线性关系，且斜 率基本一致, 表明其ORR反应遵循一级动力学原 理, 不同电位下单位氧分子所转移的电子数相当 35 。

氧还原反应是一个复杂的电化学过程, 其反 应历程分为直接四电子途径 (生成物只有 $\mathrm{H}_{2} \mathrm{O}$ 或 $\mathrm{OH}^{-}$，不产生中间产物)和二电子途径(中间产物 $\mathrm{H}_{2} \mathrm{O}_{2}$ 或 $\mathrm{HO}_{2}^{-}$, 其强氧化性严重影响催化剂和电极 的使用寿命)两种 32 。因此, 对于燃料电池和金属空气电池来说, 直接四电子途径是最理想的。图6a 为 ALD50-Pt/TiN 在 $0.5 \mathrm{~mol} \cdot \mathrm{L}^{-1} \mathrm{H}_{2} \mathrm{SO}_{4}$ 溶液中 1600 $\mathrm{r} \cdot \mathrm{min}^{-1}$ 转速下的盘电流和环电流, 可进一步计算

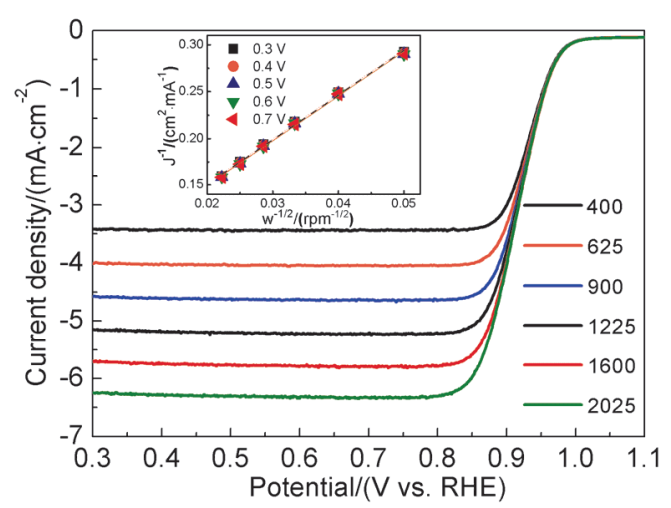

图 5 ALD-Pt/TiN 在氧气饱和的 $0.5 \mathrm{~mol} \cdot \mathrm{L}^{-1} \mathrm{H}_{2} \mathrm{SO}_{4}$ 溶液中的线性扫描伏安曲线和 K-L 曲线图(插图), 转速为 400-2025 r. $\mathrm{min}^{-1}$, 扫描速率为 $10 \mathrm{mV} \cdot \mathrm{s}^{-1}$

Fig. 5 LSV curves and related K-L plots (insert) of ALD-Pt/TiN in $\mathrm{O}_{2}$-saturated $0.5 \mathrm{~mol} \cdot \mathrm{L}^{-1} \mathrm{H}_{2} \mathrm{SO}_{4}$ solution at $10 \mathrm{mV} \cdot \mathrm{s}^{-1}$ scan rate and different rotation rates from 400 to $2025 \mathrm{r} \cdot \mathrm{min}^{-1}$. 

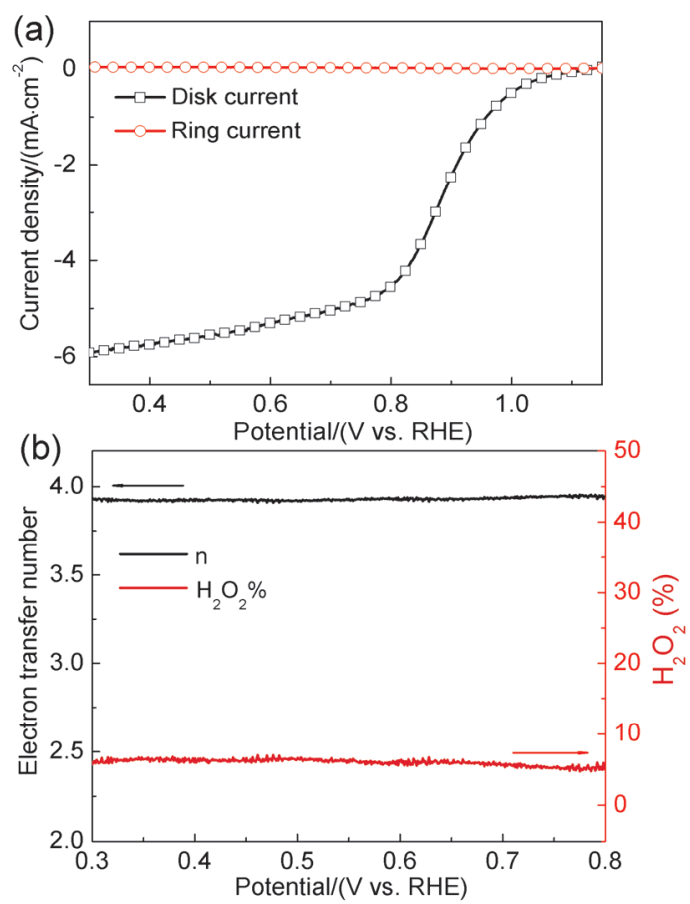

图 $6 \mathrm{ALD50-Pt/TiN}$ 的 RRDE 曲线(a)和相对应的 转移电子数(黑色)、 $\mathrm{H}_{2} \mathrm{O}_{2}$ 生成率(红色) (b)

Fig. 6 RRDE voltammograms of ALD50-Pt/TiN (a) and the corresponding Electron transfer number (black) and $\mathrm{H}_{2} \mathrm{O}_{2}$ production plots (red) (b).

确定ALD50-Pt/TiN在ORR过程中的电子转移数和 过氧化氢的生成率 ${ }^{36}$ (详见图S3 (SI))。由图6b可 知, ALD50-Pt/TiN催化剂ORR的电子转移数约为 3.93 , 表明电极上的氧气还原反应以高效的四电子 途径为主导, 过氧化氢等副产物生成较少, 仅为 $5 \%$ 。

对于ORR催化剂来说, 良好的稳定性和耐久 性对实际应用有着重要意义。图7为ALD50-Pt/TiN

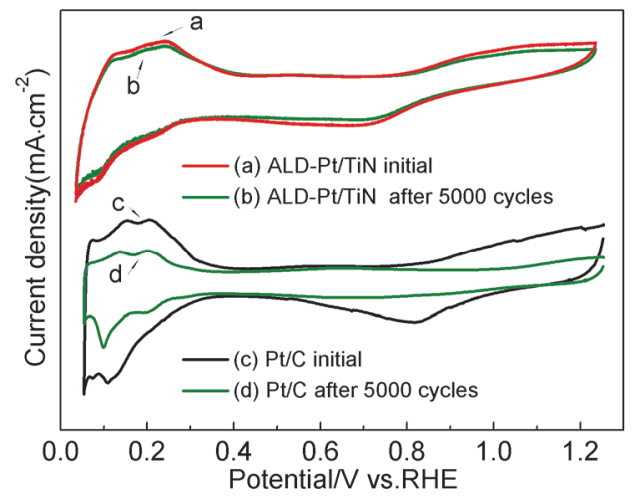

图 7 ALD50-Pt/TiN 和 Pt/C 经过 5000 圈 ADT 测试的 $\mathrm{CV}$ 曲线

Fig. 7 CV curves after 5000 cycles for ALD50-Pt/TiN and $\mathrm{Pt} / \mathrm{C}$.
和商用 $\mathrm{Pt} / \mathrm{C}$ 经过 5000 圈 $\mathrm{ADT}$ 测试的 $\mathrm{CV}$ 曲线。对比 发现, 经过5000圈加速衰减测试后 $\mathrm{Pt} / \mathrm{C}$ 催化剂的氢 区面积有较大的损失。相对 $\mathrm{Pt} / \mathrm{C}$ 而言, ALD50$\mathrm{Pt} / \mathrm{TiN}$ 表现出更好的ORR稳定性。推测主要归因两 个作用: 一方面, TiN具有良好的抗腐蚀性能, 部 分缓解了因载体腐蚀带来的Pt颗粒迁移、脱落等 问题 ${ }^{36-38}$ 。但经ADT测试后, 负载在 $\mathrm{TiN}$ 上的 $\mathrm{Pt}$ 仍 存在一定的迁移和聚集现象(详见图S3(SI)); 另一 方面, ALD50-Pt/TiN之间强的相互作用, 改变了 Pt 原子的电子结构, 使 $\mathrm{Pt}$ 的氧化变得更难, 进而增强 了Pt的稳定性 16,27 。

\section{4 结论}

本文使用具备高比表面积、抗腐蚀性好、一维 多孔结构的TiN材料作为载体, 采用原子层沉积技 术在其上制备出Pt纳米颗粒高度分散、与载体相 互作用强的 $\mathrm{Pt} / \mathrm{TiN}$ 复合材料。TEM和XRD结果表 明, TiN表面负载的Pt纳米颗粒尺寸分布均匀、主 要为面心立方晶格。XPS数据显示ALD-Pt/TiN中Pt $4 f$ 的结合能相对 $\mathrm{Pt} / \mathrm{C}$ 发生 $0.33 \mathrm{eV}$ 的正移, 说明 ALD-Pt纳米颗粒与 TiN载体间存在强的电子相互 作用。氧还原性能和稳定性测试表明, ALD-Pt/TiN 的稳态极限电流、起始电位与商用 $\mathrm{Pt} / \mathrm{C}$ 相近, 其半 波电位则高出 $20 \mathrm{mV}$, 同时兼具良好的稳定性和耐 久性。ALD-Pt/TiN复合材料优良的电催化性能主 要来源于ALD沉积Pt纳米颗粒的高分散性、TiN特 殊的一维多孔管状结构以及ALD-Pt与 TiN载体间 较强的相互作用等多方面共同作用。该工作为设 计新型高催化活性、高稳定的电催化剂提供了思 路。后续研究会进一步完善ALD-Pt与 $\mathrm{TiN}$ 载体间较 强的相互作用等方面的研究。

Supporting Information: available free of charge via the internet at http://www.whxb.pku.edu.cn.

\section{References}

(1) Lee, J. S.; Kim, S.T.; Cao, R.; Choi, N. S.; Liu, M.; Lee, K. T.; Cho, J. Adv. Energy Mater. 2011, 1, 34. doi: 10.1002/aenm. 201000010

(2) Wu, G.; More, K. L.; Johnston, C. M.; Zelenay, P. Science 2011, 332, 443. doi: $10.1126 /$ science. 1200832

(3) Dai, L.; Xue, Y.; Qu, L.; Choi, H. J.; Baek, J. B. Chem. Rev. 2015, 115, 4823. doi: 10.1021/cr5003563

(4) Van Pham, C.; Klingele, M.; Britton, B.; Vuyyuru, K. R.; Unmuessig, T.; Holdcroft, S.; Fischer, A.; Thiele, S. Adv. Sustainable Syst. 2017, 1, 1600038. doi: 10.1002/adsu.201600038

(5) Lee, J. S.; Nam, G.; Sun, J.; Higashi, S.; Lee, H. W.; Lee, S.; Chen, 
W.; Cui, Y.; Cho, J. Adv. Energy Mater. 2016, 6, 1601052. doi: 10.1002/aenm.201601052

(6) Wang, B.; Cui, X.; Huang, J.; Cao, R.; Zhang, Q. Chin. Chem. Lett. 2018, 29, 1757.doi:10.1016/j.cclet.2018.11.021

(7) Cheng, N.; Li, H.; Li, G.; Lv, H.; Mu, S.; Sun, X.; Pan, M. Chem. Commun. 2011, 47, 12792. doi: 10.1039/C1CC15203C

(8) Stephens, I. E.; Bondarenko, A. S.; Gronbjerg, U.; Rossmeisl, J.; Chorkendorff, I. Energy Environ. Sci. 2012, 5, 6744. doi: 10.1039/C2EE03590A

(9) Ramli, Z.; Kamarudin, S. K. Nanoscale Res. Lett. 2018, 13, 410. doi: 10.1186/s11671-018-2799-4

(10) Antolini, E. Int. J. Energy Res. 2018, 42, 3747. doi :10.1002/er.4134

(11) Luo, M. C.; Sun, Y. J.; Qin, Y. N.; Yang, Y.; Wu, D.; Guo, S. J. Acta Phys. -Chim. Sin. 2018, 34, 361. [骆明川, 孙英俊, 秦英楠, 杨勇, 吴冬, 郭少军. 物理化学学报, 2018, 34, 361.] doi: 10.3866/PKU.WHXB201708312

(12) Chang, Q.W.; Xiao, F.; Xu, Y.; Shao, M. H. Acta Phys. -Chim. Sin 2017, 33, 9. [常乔婉, 肖菲, 徐源, 邵敏华. 物理化学学报, 2017, 33, 9.] doi: 10.3866/PKU.WHXB201609202

(13) Sui, S.; Wang, X.; Zhou, X.; Su, Y.; Riffat, S.; Liu, C. J. J. Mater. Chem. A 2017, 5, 1808. doi:10.1039/C6TA08580F

(14) Khalily, M. A.; Patil, B.; Yilmaz, E.; Uyar, T. Nanoscale Adv. 2019, 55, 1225. doi: 10.1039/C8NA00330K

(15) Hsu, I. J.; Hansgen, D. A.; McCandless, B. E.; Willis, B. G.; Chen, J. G. J. Phys. Chem. C 2011, 115, 3709. doi: 10.1021/jp111180e

(16) Cheng, N.; Banis, M. N.; Liu, J.; Riese, A.; Mu, S.; Li, R.; Sham, T. K.; Sun, X. Energy Environ. Sci. 2015, 8, 1450. doi: 10.1039/C4EE04086D

(17) Shu, T.; Liao, S. J.; Hsieh, C. T.; Roy, A. K.; Liu, Y. Y.; Tzou, D. Y.; Chen, W. Y. Electrochim. Acta 2012, 75, 101. doi: 10.1016/j.electacta.2012.04.084

(18) Dasgupta, N. P.; Liu, C.; Andrews, S.; Prinz, F. B.; Yang, P. J. Am. Chem. Soc. 2013, 135, 12932. doi: 10.1021/ja405680p

(19) Zhang, J.; Yu, Z.; Gao, Z.; Ge, H.; Zhao, S.; Chen, C.; Chen, S.; Tong, X.; Wang, M.; Zheng, Z.; et al. Angew. Chem. Int. Ed. 2017, 56, 816. doi: 10.1002/anie201611137

(20) Cargnello, M.; Doan Nguyen, V. V.; Gordon, T. R.; Diaz, R. E.; Stach, E. A.; Gorte, R. J. Science 2013, 341, 771. doi: $10.1126 /$ science. 1240148

(21) Tian, X. L.; Luo, J.; Nan, H.; Zou, H.; Chen, R.; Shu, T.; Li, X.; Li, Y.; Song, H.; Liao, S. J. Am. Chem. Soc. 2016, 138, 1575. doi: $10.1021 /$ jacs.5b11364
(22) Ottakam Thotiyl, M.O.; Ravikumar, T.; Sampath, S. J. Mater. Chem. 2010, 20, 10643. doi: 10.1039/C0JM01600D

(23) Yang, M.; Cui, Z.; Di Salvo, F. J. Phys. Chem. Chem. Phys. 2013, 15, 1088. doi: 10.1039/C2CP44215A

(24) Wang, Y. J.; Wilkinson, D. P.; Zhang, J. Chem. Rev. 2011, 111, 7625. doi: $10.1021 / \mathrm{cr} 100060 \mathrm{r}$

(25) Xiao, Y.; Zhan, G.; Fu, Z.; Pan, Z.; Xiao, C.; Wu, S.; Chen, C.; Hu, G.; Wei, Z. J. Power Sources 2015, 284, 296. doi: 10.1016/j.jpowsour.2015.03.001

(26) Luo, J.; Tang, H.; Tian, X.; Hou, S.; Li, X.; Du, L.; Liao, S. ACS Appl. Mater. Interface 2018, 10, 3530. doi: 10.1021/acsami.7b15159

(27) Shin, H.; Kim, H. I.; Chung, D. Y.; Yoo, J. M.; Weon, S.; Choi, W.; Sung, Y. E. ACS Catal. 2016, 6, 3914. doi: $10.1021 /$ acscatal.6b00384

(28) Zhu, X.; Yang, X.; Lv, C.; Guo, S.; Li, J.; Zheng, Z.; Zhu, H.; Yang, D. ACS Appl. Mater. Interfaces 2016, 8, 18815. doi: 10.1021/acsami.6b04588

(29) Li, C.; Tan, H.; Lin, J.; Luo, X.; Wang, S.; You, J.; Kang, Y. -M.; Bando, Y.; Yamauchi, Y.; Kim, J. Nano Today 2018, 21,91. doi: 10.1016/j.nantod.2018.06.005

(30) Zhang, N.; Zhang, S.; Du, C.; Wang, Z.; Shao, Y.; Kong, F.; Lin, Y.; Yin, G. Electrochim. Acta 2014, 117, 413. doi: 10.1016/j.electacta.2013.11.139

(31) Seifitokaldani, A.; Savadogo, O.; Perrier, M. Electrochim. Acta 2014, 141, 25. doi:10.1016/j.electacta.2014.07.027

(32) Nie, Y.; Li, L.; Wei, Z. Chem. Soc. Rev. 2015, 44, 2168. doi: $10.1039 / \mathrm{C} 4 \mathrm{CS} 00484 \mathrm{~A}$

(33) Bai, Q.; Shen, F. C.; Li, S. L.; Liu, J.; Dong, L. Z.; Wang, Z. M.; Lan, Y. Q. Small Methods 2018, 2, 1800049. doi: $10.1002 / \operatorname{smtd} .201800049$

(34) Liu, C.; Wang, J.; Li, J.; Liu, J.; Wang, C.; Sun, X.; Shen, J.; Han, W.; Wang, L. J. Mater. Chem. A 2017, 5, 1211. doi:10.1039/C6TA09193H

(35) Marković, N. M.; Schmidt, T. J.; Stamenković, V.; Ross, P. N. Fuel Cells 2001, 1, 105. doi: 10.1002/1615-6854(200107)

(36) Zhao, Y.; Lai, Q.; Zhu, J.; Zhong, J.; Tang, Z.; Luo, Y.; Liang, Y. Small 2018, 14, 1704207. doi: 10.1002/smtd.201800049

(37) Kulkarni, A.; Siahrostami, S.; Patel, A.; Nørskov, J. K. Chem. Rev. 2018, 118, 2302. doi: 10.1021/acs.chemrev.7b00488

(38) Cui, Z.; Yang, M.; Chen, H.; Zhao, M.; DiSalvo, F. J. ChemSusChem 2014, 7, 3356. doi: 10.1002/cssc. 201402726 